\title{
El modo de proceder de Fe y Alegría
}

\section{Antonio Dan Narváez-Coronel ${ }^{20}$}

Fe y Alegría, Ecuador

a.narvaez@feyalegria.org.ec

Artículo recibido en noviembre y aprobado en diciembre 2020

\section{Resumen}

En los últimos años, Fe y Alegría ha hecho referencia a su modo de proceder, expresión que denota las cualidades distintivas de una institución $y$, en consecuencia, aporta al mejoramiento de la cultura organizacional, favoreciendo el vínculo de las colaboradoras y colaboradores con el propósito institucional, trascendiendo las clásicas relaciones contractuales. En la tradición ignaciana, de la que Fe y Alegría participa, el modo de proceder se remonta a las Constituciones de la Compañía de Jesús. En este sentido, una caracterización del modo de proceder de Fe y Alegría requiere, hasta cierto punto, referirse al de la Compañía de Jesús. El ensayo revisará, en primer lugar, el modo de proceder de la Compañía y, en un segundo momento, el de Fe y Alegría, previo un recorrido por los documentos de los congresos internacionales que la Federación Internacional realiza anualmente. Se concluye, entre otros puntos, que el modo de proceder de Fe y Alegría impulsa a practicar lo que promueve: la disponibilidad, el desprendimiento, la entrega a las personas en situación de pobreza y la coherencia.

Palabras clave: modo de proceder, cultura organizacional, identidad de Fe y Alegría, Compañía de Jesús.

\section{The way to proceed of Fe y Alegría}

\section{Abstract}

In recent years, Fe y Alegría has made reference to its way to proceed, an expression that indicates the distinctive qualities of

20 Máster en Diseño y Gestión de Proyectos Socioeducativos (Universidad Central del Ecuador), Diplomado Superior en Investigación Social (CLACSO). Coordinador de Planificación y Gestión Estratégica de Fe y Alegría Ecuador 
an institution and, consequently, contributes to the improvement of the organizational culture, encouraging the ideal affinity between collaborators and the institutional purpose, transcending the classic contractual relations. In the Ignatian tradition, in which Fe y Alegría participates, the way to proceed goes back to the Constitutions of the Society of Jesus. In this sense, a characterization of the way to proceed of Fe y Alegría requires, to a certain extent, reference to that of the Society of Jesus. The essay will first review the way to proceed of the Society of Jesus and, then, Fe y Alegría's, after a review of the documents of the international congresses that the International Federation holds annually. It is therefore concluded, among other points, that the way to proceed of Fe y Alegría encourages the practice of what it promotes: availability, detachment, dedication to disadvantaged people and coherence.

Keywords: way to proceed, organizational culture, Identity of Fe y Alegría, Society of Jesus.

\section{Introducción}

La referencia al modo de proceder de Fe y Alegría se ubica en el ámbito de la cultura organizacional; implica comportamientos, valores, modos de ser, etc. (Goleman, D. et al, 2015), e involucra una relación con el Movimiento que va más allá del vínculo contractual; supone un propósito misional de la organización que forma parte del proyecto de vida de los colaboradores y colaboradoras, enriqueciéndose mutuamente, sin detrimento de ninguna de las partes (FIFyA, 2010). En este sentido, el modo de proceder de Fe y Alegría se alinea con la conceptualización de "Fraternidad y amistad social", propuesta por el papa Francisco en la encíclica Frateli Tutti (2020) porque no alude al "ganar-ganar" propio de los socios conforme los criterios neoliberales del libre mercado.

En Fe y Alegría, el modo de proceder sugiere cualidades intrínsecas que lo caracterizan como Movimiento de Educación Popular y lo diferencian de otras instituciones; en cuanto, obra de la Compañía de Jesús, la referencia al modo de proceder de la Orden ofrece luces para comprender el de Fe y Alegría.

El método que se empleará en el presente ensayo es inductivo. 
Se recorrerán las experiencias recogidas en documentos institucionales, para luego formular las características del modo de proceder del Movimiento y manifestar algunas conclusiones.

\section{El modo de proceder en la Compañía de Jesús}

En la tradición ignaciana, la referencia al modo de proceder alude a cualidades propias de la Compañía de Jesús y se remonta a sus Constituciones. Con los siglos, la expresión se ha matizado según el contexto de la Iglesia y del mundo en que la Orden ha desarrollado su actividad apostólica. Una de las revisiones más amplias la desarrolló el padre Pedro Arrupe, general de la Compañía de Jesús (1965 - 1983), en la conferencia "El modo nuestro de proceder" que pronunció en el Centro Ignaciano de Espiritualidad de Roma, en 1979; posteriormente, el modo de proceder ha sido matizado en ulteriores congregaciones generales (Mollá, 2015). A continuación, se conceptualizará el significado del modo de proceder y las posibles implicaciones en la caracterización del modo de proceder de Fe y Alegría.

\section{El modo de proceder en las Constituciones de la Compañía de Jesús}

En las Constituciones, la referencia al modo de proceder es relativamente habitual:

- "conforme a la nuestra profesión y modo de proceder, para discurrir por unas partes y por otras del mundo" (Constituciones, Examen, $N^{\circ} 1,92$ ).

- "Suele ser conveniente modo de proceder de lo menos a lo más perfecto, en especial para la práctica" (Constituciones, $\left.N^{\circ} 1,137\right)$.

- " teniendo discreción y modo de proceder, con tan diversas maneras y condiciones de personas" (Constituciones, $N^{0} 1$, 142).

El padre Arrupe indica que en las Constituciones se encuentran no menos de 16 referencias al modo de proceder y que apuntan al 
"conjunto de originalidades, notas específicas y diferenciales de la Compañía respecto de las contemporáneas órdenes religiosas... en abierto contraste con los [religiosos] habituales de la época" (Arrupe, 1979. p. 190).

Para dimensionar la importancia del "contraste" con los religiosos de la época es necesario contextualizar el momento histórico del S. XVI en que surge la Orden: los ideales del Humanismo, el Renacimiento, la ebullición de la Reforma Protestante y, por otra parte, la preocupación desmesurada de la Iglesia por los asuntos temporales, la confusión doctrinal de los fieles y el ambiente de corrupción que permeaba varios niveles eclesiales (Ruiz-Doménec, 2013). En este sentido, el modo de proceder de la Compañía de Jesús puede considerarse una toma de postura crítica frente a las situaciones de degradación eclesial de la época, que favorece una regeneración desde dentro y sin rupturas al estilo de los reformadores protestantes.

\section{El modo de proceder en las congregaciones generales de la Compañía de Jesús}

a) La Congregación General 34 mantiene, en lo fundamental, el sentido y la caracterización del modo de proceder expuesto por el P. Arrupe. Desarrolla una definición que se volverá referencial en ulteriores reflexiones. Lo entiende como "actitudes, valores y patrones de conducta que, juntas, forman lo que se ha llamado el modo de proceder de la Compañía. Las características de nuestro modo de proceder nacieron en la vida de San Ignacio y fueron compartidas por sus primeros compañeros" (CG 34, Decr. 26, $n^{\circ} 1$ ).

b) La Congregación General 35 se refiere en varias ocasiones al modo de proceder de la Compañía. En el decreto 2, "Un fuego que enciende otros fuegos. Redescubrir nuestro carisma", le dedica especial atención y lo entiende como el "descubrir las huellas de Dios en todas partes" (Decr. 2, n 8). Además, pide que los colaboradores no jesuitas se formen en el modo de proceder de la Compañía: "Los superiores mayores, al nombrar directores de obra que no sean jesuitas, atiendan ... a su comprensión y a su compromiso con nuestra misión y modo de 
proceder" (Decr. 4, n48), y explicita que la formación de directivos de obras "deben recibir una formación adecuada en lo propio de nuestro modo de proceder, especialmente la integración del discernimiento apostólico" (Decr. 6, n²0).

c) La Congregación General 36 indica que los "Modos de proceder apropiados para nuestro tiempo" son "El discernimiento, la colaboración y el trabajo en red" (Decr. 2, n³), y particularmente invita a jesuitas y colaboradores en la misión, "a promover profundos hábitos de oración y discernimiento como preludio y acompañamiento de la planificación ... a fortalecer las relaciones interpersonales y la colaboración en la implementación de los planes... Todo esto implica fomentar un espíritu de disponibilidad y confianza entre nosotros y con todos aquellos que sirven a la Missio Dei." (Decreto 2, $n^{\circ} 26$ ).

Este breve recorrido por el modo de proceder de la Compañía de Jesús, muestra que se refiere a cualidades propias de la Orden, que la identifican, tanto en el contexto de la época de su fundación, como en la actualidad. También se puede apreciar que, si bien en los inicios, el modo de proceder se delimitaba a los jesuitas, en las últimas décadas, la Compañía de Jesús lo propone a las colaboradoras y colaboradoras de sus obras, particularmente el discernimiento en común, la colaboración en redes y la disponibilidad apostólica. Estas cualidades también conformarán el modo de proceder de Fe y Alegría.

\section{El modo de proceder de Fe y Alegría}

Fe y Alegría, en cuanto Movimiento de Educación Popular Integral y de Promoción Social, ha configurado un modo de proceder propio; para caracterizarlo se buscará su rastro en los documentos de los Congresos Internacionales de la Federación Internacional, pues ellos recogen la reflexión y los derroteros de Fe y Alegría en los países donde sirve. 


\section{El modo de proceder de Fe y Alegría según los congresos internacionales}

a) En el XXXVII Congreso (2007), "Nuestra práctica de promoción social comunitaria", se dice:

"Nuestro modo de proceder tiene que comenzar a construir esa sociedad nueva, más solidaria y tolerante, capaz de afirmar su identidad en diálogo no sólo de palabras, sino de voluntades y acciones, con la diversidad. En la tarea de promoción comunitaria tenemos nuestro principal reto de aprender a trabajar en equipo, a construir liderazgos compartidos, a fortalecer las actitudes y estructuras de la democracia participativa. La calidad de nuestro trabajo de promoción, como la del educativo, no se mide sólo por los resultados, sino también por los procesos, que atentos al contexto en que se mueve, construyen un modo de proceder que se inspira y responde a los valores que queremos construir" (FIFyA. 2008. p. 35).

b) En el XL Congreso Internacional (2009), "Hacer el bien y hacerlo bien", en el contexto de la explicitación de las características de la gestión de Fe y Alegría, en el Documento Base, en relación al modo de proceder de Fe y Alegría, se afirma que:

- "La autonomía funcional, más que una parcela de poder debe ser entendida como un modo de proceder en la misión compartida" (FIFyA. 2010. p. 35), afirmación que se confirma en la reacción de Joseba Lazcano a la presentación de Víctor Murillo sobre Autonomía funcional, redes, construcción organizativa, descentralización: "Por eso, la autonomía funcional que hoy quiere ser, con agradecimiento, fiel al fundador será expresión de espiritualidad... Por eso, la autonomía funcional, más que parcela de poder, será modo de proceder" (FIFyA. 2010. p. 82), insistiendo que "la autonomía funcional de Fe y Alegría es -seguirá siendoexpresión de su espiritualidad" (FIFyA. 2010. p. 83).

- "Estructuralmente, la identidad da razón de ser a su modo de 
proceder, a sus elementos esenciales y a sus características fundamentales" (Orbegozo. 2002. Cit. por FIFyA. 2010. p. 46), que implica: "definir políticas de formación permanente y organizar procesos de formación que garanticen el estilo de gestión y el modo de proceder de Fe y Alegría" (FIFyA. 2010. p. 51), "fortalecer el trabajo en red, potenciando nuevas estructuras organizativas ... a nivel interno y para poder participar en redes sociales externas, y las tecnologías de la información y las comunicaciones, como medios para que la cultura democrática y colaborativa sea una realidad y haga parte de la "personalidad", del modo de proceder del Movimiento" (FIFyA. 2010. p. 53).

c) En el XLII Congreso de la Federación Internacional de Fe y Alegría (2011), "Identidad y Espiritualidad al servicio de la misión":

- El documento de trabajo "Identidad y espiritualidad al servicio de la misión", al referirse a la identidad de Fe y Alegría, expresa que "brota de y se manifiesta en una espiritualidad encarnada que se esfuerza por testimoniar en todo su quehacer, y en su modo de proceder, la misión y los valores que proclama" (FIFyA. 2012. p. 11). Más adelante, al esbozar los rasgos de la espiritualidad de Fe y Alegría, entre otros, destaca los siguientes asociados al modo de proceder del Movimiento:

- "Espiritualidad maternal que ama, celebra y defiende toda forma de vida ... que reivindica la igualdad de la mujer y la construcción de políticas y prácticas de equidad de género... combate las estructuras y cultura machista y patriarcal, tan extendidas todavía en nuestras sociedades y en la propia Iglesia. Afirmar esto debe llevarnos también en Fe y Alegría a revisar nuestras estructuras de poder, nuestro trato y modo de proceder" (FIFyA. 2012. p. 31).

- Además, indica que otro rasgo de la espiritualidad de Fe y Alegría "se traduce y concreta en nuestro modo de 
proceder... es, en definitiva, una espiritualidad del amor práctico y eficaz: es encontrar a Dios en el hermano, sobre todo el más desvalido y necesitado" (FIFyA. 2012. p. 32).

- El documento "La espiritualidad ignaciana ilumina la espiritualidad de Fe y Alegría", al referirse a las experiencias fundantes de la espiritualidad de la Compañía de Jesús, el padre Ernesto Cavassa, S. J. se refiere en varias ocasiones al modo de proceder de la Orden (Cfr. FIFyA. 2012, p. 71-76). Más adelante, se pregunta por el modo en que la espiritualidad ignaciana ilumina la de Fe y Alegría. Una de las opciones que considera es "comparar los rasgos del modo de proceder ignaciano ... y aplicarlo a Fe y Alegría". Sin embargo, se inclina por explorar en "la espiritualidad ignaciana y la de Fe y Alegría, algunos puntos en común" (FIFyA. 2012. p. 76). El primero de los rasgos constituye "La primacía del fundador, como primer testigo" que, en el caso de Ignacio, en palabras del padre Nadal, representa "un ejemplo vivo de nuestro modo de proceder" (FIFyA. 2012. p. 77). Otro rasgo lo describe como "Una espiritualidad convocante y fecunda, de colaboración", donde cae en cuenta que el decreto 6, "Colaboración en el corazón de la misión", de la Congregación General 35 menciona

"explícitamente a Fe y Alegría ... y concluye diciendo que "la colaboración es una gracia que se nos regala en este momento en perfecta coherencia con nuestro modo jesuita de proceder". Fe y Alegría es mencionada como una red, obra de la Compañía," (FIFyA. 2012. p. 83; CG 35. Decr. 6, N²2)

- En otro documento del Congreso, "Algunas notas sobre la identidad de Fe y Alegría", Antonio Pérez Esclarín, a modo de motivación introductoria, expresa que el avanzar de Fe y Alegría

"Es un caminar tras las huellas de Jesús... contra corriente ... muy distinto a los criterios de las 
empresas e instituciones "exitosas", con unos valores determinados, un modo de proceder radicalmente distinto, que encarna en la conducta y en la práctica los valores que promueve" (FIFyA.2012. p. 102).

Este caminar se distingue por la

"Indiferencia: es decir, no estar apegados a cargos, obras ... Vivir disponibles, con un pie alzado, en actitud de éxodo permanente, abandonando ... estilos y modos de proceder que hemos hecho parte de nuestra cultura y de nuestra vida y que incluso llegamos a considerar como "derechos adquiridos" (FIFyA. 2012. p. 102).

Reitera que

"En cuanto al modo de proceder de nuestros centros de trabajo ... deben expresar los valores que predicamos (sencillez, austeridad, amistad, solidaridad, trabajo responsable, servicio, fe, alegría, optimismo, esperanza...). La idea es que se vayan constituyendo en pequeños microcosmos de esa nueva sociedad que pretendemos" (FIFyA. 2012. p. 103).

d) En el XLVII Congreso Internacional, (2018), "Educamos en las Fronteras, Fe y Alegría, Movimiento Global", el Padre General de la Compañía de Jesús, Arturo Sosa, pronuncia el discurso "Educamos en las Fronteras" donde hizo mención al modo de proceder, tanto en relación a la Compañía de Jesús, como a Fe y Alegría.

Acerca del trabajo en red, en referencia a la Compañía de Jesús, el Padre General expresa que "Esa es la razón por la cual la CG 36 (D2, 8) insiste en el trabajo en red como modo de proceder privilegiado para hacer más universal el servicio de la Compañía" (Sosa, A. 2018. p. 3) Recalca que "El trabajo en red es una de las intuiciones más poderosas que hemos ido vislumbrando en estas últimas décadas que se ha 
convertido en una dimensión central del modo nuestro de proceder" (Sosa, A. 2018. p. 4).

A propósito del trabajo en red de Fe y Alegría considera

"muy sugerente y audaz la propuesta de renovación de su modo de proceder, a través del cambio de los programas federativos a la nube de redes, buscando una mayor implicación y participación de los países, impulsando la comunicación y, especialmente, ayudando a gestar un nuevo modo de liderazgo más abierto, inclusivo y dinamizador" (Sosa, A. 2018. p. 4).

Además del trabajo en red, motiva a la vivencia del discernimiento como rasgo del modo de proceder de Fe y Alegría: "Si incorporan el discernimiento en común en su modo ordinario de proceder podrán desarrollar la sensibilidad necesaria para encontrar dónde el Señor les pide estar" (Sosa, A. 2018. p. 10).

\section{El modo de proceder de Fe y Alegría: características a partir de los congresos internacionales}

1. Trabajo en equipo (FIFyA. 2008. p. 35). FIFyA. 2010. p. 53

2. Liderazgos compartidos, más abiertos, inclusivos y dinamizadores, con estructuras democráticas (FIFyA. 2008. p. 35). (Sosa, A. 2018. p. 4).

3. Autonomía funcional (FIFyA. 2010. p. 35, 82, 83).

4. Formación permanente (FIFyA. 2010. p. 51),

5. Trabajo en redes externas (FIFyA. 2010. p. 53; FIFyA. 2012. p. 83; CG 35. Decr. 6, N²2; CG 36, Decr. 2, $n^{\circ} 3$ ).

6. Que camina tras las huellas de Jesús, contracorriente de las empresas e instituciones exitosas (FIFyA. 2012. p. 102) 
7. Disponibilidad, con un pie alzado, en actitud de éxodo permanente (FIFyA. 2012. p. 102).

8. Que expresa los valores que predicamos (sencillez, austeridad, amistad, solidaridad, trabajo responsable, servicio, fe, alegría, optimismo, esperanza) como testimonio de la sociedad que promovemos (FIFyA. 2012. p. 103).

9. Que incorpora el discernimiento como rasgo del modo de proceder de Fe y Alegría (Sosa, A. 2018. p. 10; CG 35, Decr. 6, $n^{\circ} 20 ; C G 36$, Decreto 2, $n^{\circ} 26$ ).

10. Maternal, que ama, celebra y defiende toda forma de vida (FIFyA. 2012. p. 31).

11. Que practica el amor práctico y eficaz, que encuentra Dios en el hermano, sobre todo en el más desvalido y necesitado (FIFyA. 2012. p. 32).

\section{Conclusiones}

a. Parafraseando las definiciones del modo de proceder que realizan el P. Arrupe (1979) y la Congregación General 34 (1995), el modo de proceder de Fe y Alegría se entiende como aquellas cualidades que configuran su personalidad, tanto en sus notas externas como en sus notas esenciales; expresan las notas propias de su identidad como Movimiento de Educación Popular. El modo de proceder de Fe y Alegría se constituye por un conjunto de originalidades, notas específicas y diferenciales respecto de otras instituciones educativas, inclusive de educación popular; lo hacen único e irrepetible, son sus actitudes, valores y patrones de conducta. Como se ha visto a lo largo de la reflexión, las características del modo de proceder del Movimiento, siguen en construcción, se han ido caracterizando conforme avanza y evoluciona en la historia.

b. Si el modo de proceder de la Compañía responde a una fusión de horizontes en los contextos donde despliega su labor apostólica, el modo de proceder de Fe y Alegría responde a la convergencia de necesidades que continúan viviendo muchos 
sectores de la población empobrecida de Latinoamérica y del mundo: vulneración de derechos, exclusión de servicios básicos, afectación por todos los daños socioambientales, etc. De esta manera, el modo de proceder de Fe y Alegría se hace eco de la opción preferencial por los empobrecidos que expresa su Ideario.

c. Aunque Fe y Alegría está precedida históricamente por la Compañía de Jesús, su modo de proceder lo desarrolla de manera autónoma, de forma independiente al de la Orden, de acuerdo a su naturaleza de Movimiento de Educación Popular, respondiendo a una configuración propia. Con todo, en las últimas décadas, ha incorporado características del modo de proceder que la Compañía de Jesús impulsa en sus obras: trabajo en redes, discernimiento comunitario, familiaridad con la espiritualidad ignaciana, etc.

d. Considerando los Congresos Internacionales, no se puede afirmar que Fe y Alegría haya procurado tematizar su modo de proceder. Sin embargo, en varias ocasiones ha definido y actualizado las características que constituyen su identidad, de forma que el modo de proceder de Fe y Alegría surge como una deriva de su identidad. Esta afirmación se puede comprobar al revisar los planes estratégicos de la Federación. Únicamente el primero menciona el modo de proceder de Fe y Alegría y lo relaciona con el proyecto 1: Fortalecimiento de la Identidad del Movimiento (FIFyA, 2000).

e. Si algo atraviesa el modo de proceder de Fe y Alegría es el impulso a servir que nace de la mirada a una humanidad que cae, se levanta y continúa; es un ser humano iluminado por el Dios de la vida, Padre y Madre de la creación que, en este mundo cada vez menos solidario, más egoísta, sin fe, no es un patrón pendiente de las pérdidas y ganancias, de los cumplimientos, de los incumplimientos, sino que considera los obstáculos que se superan, los procesos, las dificultades vencidas que representan la lucha del creyente en su opción por la misión de Dios.

f. El modo de proceder de Fe y Alegría busca la coherencia entre su ser institucional y la sociedad que promueve. Si busca 
una sociedad más participativa, democrática, horizontal, pide que, antes, se revisen sus estructuras internas de gobierno. Si promueve el manejo óptimo de los recursos, la sencillez y la austeridad, así lo suscita en sus centros educativos. Si estimula para que la sociedad se preocupe por las personas vulneradas en sus derechos, al empezar donde termina el asfalto, Fe y Alegría da el primer paso. Con estas pequeñas acciones, que se reflejan en su modo de proceder, Fe y Alegría quiere seguir las huellas de Jesús de Nazareth y contribuye para que nuestro mundo sea más justo, más humano, más socialmente fraterno.

\section{Referencias}

Arrupe, P. (1979). "El modo nuestro de proceder" (Conferencia del P. Pedro Arrupe en el Curso Ignaciano del Centro Ignaciano de Espiritualidad de Roma el 18 de enero de 1979). En: Mollá, D. (2015). Pedro Arrupe, carisma de Ignacio. Madrid: Mensajero-Sal Terrae. p. 185-232.

Compañía de Jesús. (1995). Congregación General 34 de la Compañía de Jesús. Tomado de: http://pedagogiaignaciana.com/GetFile. ashx?ldDocumento $=521$

Compañía de Jesús. (2008). Congregación General 35 de la Compañía de Jesús. Tomado de: http://www.sjweb.info/35/documents/ decretos.pdf

Federación Internacional de Fe y Alegría (FIFyA). (2001). PLAN GLOBAL DE DESARROLLO Y FORTALECIMIENTO INSTITUCIONAL 20012005. Caracas: Federación Internacional Fe y Alegría.

Federación Internacional de Fe y Alegría (FIFyA). (2008). Revista Internacional Fe y Alegría $N^{\circ}$ 9. Nuestra práctica de promoción social comunitaria. Caracas: Federación Internacional Fe y Alegría.

Federación Internacional de Fe y Alegría (FIFyA). (2010). Revista Internacional Fe y Alegría $N^{0} 11$. Hacer el bien y hacerlo bien. Bogotá: Federación Internacional Fe y Alegría.

Federación Internacional de Fe y Alegría (FIFyA). (2012). Revista 
Internacional Fe y Alegría $N^{0}$ 13. Identidad y espiritualidad al servicio de la misión. Bogotá: Federación Internacional Fe y Alegría.

Goleman, D., Boyatzis, R., McKee, A. (2015). El líder resonante crea más. Buenos Aires: Sudamericana.

Jesuitas Ecuador. (2016). Congregación General 36. Quito: Secretariado de Espiritualidad

Loyola, I. (1554). Constituciones de la Compañía de Jesús. Tomado de: http://pedagogiaignaciana.com/GetFile. ashx?IdDocumento $=2909$

Mollá, D. (2015). Pedro Arrupe, carisma de Ignacio. Madrid: MensajeroSal Terrae.

Orbegozo, J. (2002), La identidad de Fe y Alegría. Notas introductorias al taller de identidad. Caracas (Mimeo.).

Ruiz-Doménec, J. (Dir). (2013). Historia, Vol. XXIII. Renacimiento y Reforma. España: National Geographic Society.

Sosa, A. (2018). Educamos en las Fronteras. Discurso del Padre General de la Compañía de Jesús. Sábado 29 de septiembre 2018. Casa San José, El Escorial, Madrid. Tomado de: https://www. congresofeyalegria2018.org/wp-content/uploads/2018/09/ Discurso-Padre-General-47-Congreso-Internacional-Fe-yAlegr\%C3\%ADa.pdf 